\title{
Estenosis aórtica crítica y síndrome de corazón izquierdo hipoplásico de diagnóstico precoz en un hospital de segundo nivel
}

\author{
Mileidy Egleet Mejias Quintero ${ }^{1}$. Violeta Román Barba ${ }^{2}$. José María Huertas González ${ }^{1}$. \\ Haidar Salem Salem ${ }^{1}$. Alberto Galindo Izquierdo. ${ }^{3}$ \\ ${ }^{1}$ Departamento de Obstetricia y Ginecología, ${ }^{2}$ Unidad de Cardiología Pediátrica, Hospital Gutiérrez Ortega de \\ Valdepeñas, Ciudad Real-España. ${ }^{3}$ Departamento de Obstetricia y Ginecología, Unidad de Medicina Fetal, Hospital \\ Universitario 12 de Octubre. Madrid-España.
}

\section{RESUMEN}

Las cardiopatías son las malformaciones congénitas más frecuentes del desarrollo prenatal, en las que se produce un defecto en la arquitectura normal del corazón fetal interfiriendo en su buen funcionamiento en la vida postnatal. La proporción entre las diferentes cardiopatías varía mucho, sin embargo el Síndrome de Corazón Izquierdo Hipoplásico representa el 2-3\% del total de cardiopatías congénitas constituyendo la forma más severa de lesiones obstructivas del corazón izquierdo y la de peor pronóstico. Su diagnóstico en etapas precoces de la gestación es hoy un reto para el obstetra, ya que su carácter evolutivo dificulta este hecho, no siendo en ocasiones, hasta etapas avanzadas de la gestación cuando se produce la alteración anatómica y la subsiguiente expresión ecográfica. Es en este punto donde se debe hacer hincapié, intentando aumentar el número de casos detectados precozmente, lo cual permitiría un adecuado asesoramiento familiar y la toma de decisiones oportuna. En los últimos años la introducción de terapias en patología cardíaca fetal, ha despertado un gran interés por tratar de modificar la evolución de esta enfermedad, siendo la valvuloplastía con balón aórtico la técnica de elección en pacientes seleccionados.

\section{PALABRAS CLAVE: Cardiopatías congénitas, diagnóstico prenatal, síndrome de corazón izquierdo hipoplásico}

\section{SUMMARY}

Congenital heart defects are the most frecuently congenital malformations in the prenatal development and consist of defect in the normal architecture of the fetal heart which interfering with its proper functioning. The proportion between the different heart disease varies widely, however Hypoplastic Left Heart Syndrome (HLHS) represent 2-3\% of all congenital heart diseases and constitute the most severe obstructive lesions of the left heart and worse prognosis. The quickly diagnosis in early stages of the disease is an obstetrician challenge, however, it's evolutional feature hinders this proposal even in advanced stages of pregnancy when the anatomical alteration and subsequent ultrasound expression can be occur. At this point that should be emphasized to try increasing the number of cases detected early, which would allow a proper family counseling and timely decision. Recent years the introduction of therapies on fetal cardiac pathology, has aroused great interest in trying to change the course of the disease and the fetal aortic valvuloplasty with aortic balloon would be the choice technique in selected patients.

KEY WORDS: Congenital heart disease, prenatal diagnosis, hypoplastic left heart syndrome 


\section{INTRODUCCIÓN}

Las cardiopatías son las malformaciones congénitas más frecuentes (1) del desarrollo prenatal, en las que se produce un defecto en la arquitectura normal del corazón fetal (2) interfiriendo en su buen funcionamiento en la vida postnatal, es por ello que su detección hoy es un objetivo prioritario. La incidencia se estima en torno $0,4-1 \%$ de recién nacidos vivos y dentro de ellas, los defectos septales son las más frecuentes (3).

La estenosis aórtica es una cardiopatía cuya incidencia real no se conoce con exactitud debido a que presenta un espectro clínico muy amplio, variando desde lesiones valvulares leves, que suelen pasar desapercibidas, hasta la forma más severa de la misma ocasionando el síndrome de corazón izquierdo hipoplásico $(\mathrm{SCIH})(4)$.

La estenosis aórtica se clasifica en función de la localización de la obstrucción en 3 tipos: valvular (60-75\%), subvalvular (8-20\%) y supravalvular (5). En la evolución de la enfermedad valvular, debido a la obstrucción severa al flujo anterógrado se produce una falta de crecimiento del ventrículo izquierdo que lo hará incapaz de mantener una adecuada circulación sistémica al nacimiento. El SCIH debe ser diagnosticado intraútero no sólo por los beneficios que el diagnóstico prenatal proporciona al paciente sino por la severidad y el mal pronóstico que conlleva el nacimiento del recién nacido en hospitales que carecen de medios suficientes para el manejo de esta cardiopatía.

El objetivo de esta comunicación es describir un caso de diagnóstico precoz de $\mathrm{SCIH}$ en nuestro Hospital que permitió la derivación de la paciente a la Unidad de Medicina Fetal, referencia en el diagnóstico prenatal de las cardiopatías congénitas.

\section{Caso Clínico}

Paciente de 36 años con una paridad de Gesta 3, Para 1, Abortos 1, con antecedentes personales de quistectomía ovárica en 2008 y cesárea en 2012 por fracaso de inducción. Presentaba un cribado del primer trimestre de bajo riesgo con una translucencia nucal de $1,2 \mathrm{~mm}$ y una PAPP-A y beta HCG normal para la edad gestacional. Serologías negativas. Acude a control del segundo trimestre de gestación encontrándose una exploración cardíaca fetal patológica.

Iniciando la exploración cardíaca fetal desde el abdomen encontramos un feto en presentación podálica, con el dorso posterior izquierdo con las vísceras abdominales localizadas correctamente. El corazón se localizaba a la izquierda con el ápex mirando hacia ese lado. La ordenación segmentaria era normal. En plano de 4 cámaras (4C) objetivamos las aurículas de tamaño normal no dilatadas con un foramen oval permeable (FOP) con flapping alterado hacia aurícula derecha (AD), posiblemente a consecuencia de la gran presión que había en la cámara subyacente, ya que llama la atención un ventrículo izquierdo (VI) de pequeño tamaño, severamente disfuncionante con hiperrefringencia marcada del subendocardio (Figura 1), como resultado de la necrosis que se origina en las células cardíacas y el depósito de fibrina secundaria a la presión suprasistémica del VI. En la evaluación del aparato valvular objetivamos una válvula mitral pequeña de apertura restrictiva que permitía un llenado ventricular monofásico (Figura 2). Con el uso del doppler color y pulsado no pudimos objetivar claramente flujo anterógrado a través de la válvula aórtica, encontrándonos ante la situación de una estenosis valvular crítica/válvula atrésica. El escaso flujo anterógrado a través del tracto de salida del VI condiciona una hipoplasia severa del arco aórtico que se debe perfundir de manera retrógrada a través del ductus arterioso (Figura 3 ), recibiendo así sangre pobre en oxígeno los troncos supraaórticos y las arterias coronarias. El resto de la exploración morfológica fetal era normal con un adecuado crecimiento y líquido amniótico.

Ante los hallazgos encontrados en la ecocardiografía fetal compatibles con $\mathrm{SClH}$ se decide derivar precozmente a la paciente a un centro terciario especializado en el diagnóstico y tratamiento de las cardiopatías congénitas, donde fue confirmado el mismo, permitiendo informar a la familia para tomar una decisión oportuna.

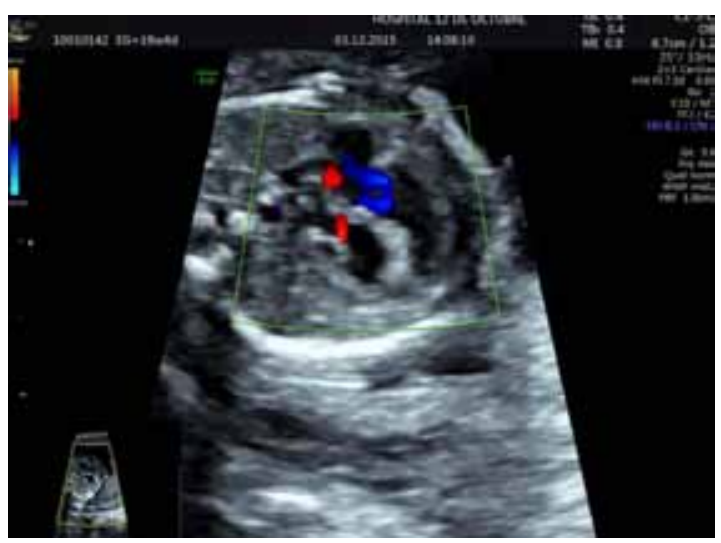

Figura 1. Ventrículo izquierdo pequeño e hiperrefringente. Insuficiencia mitral. 


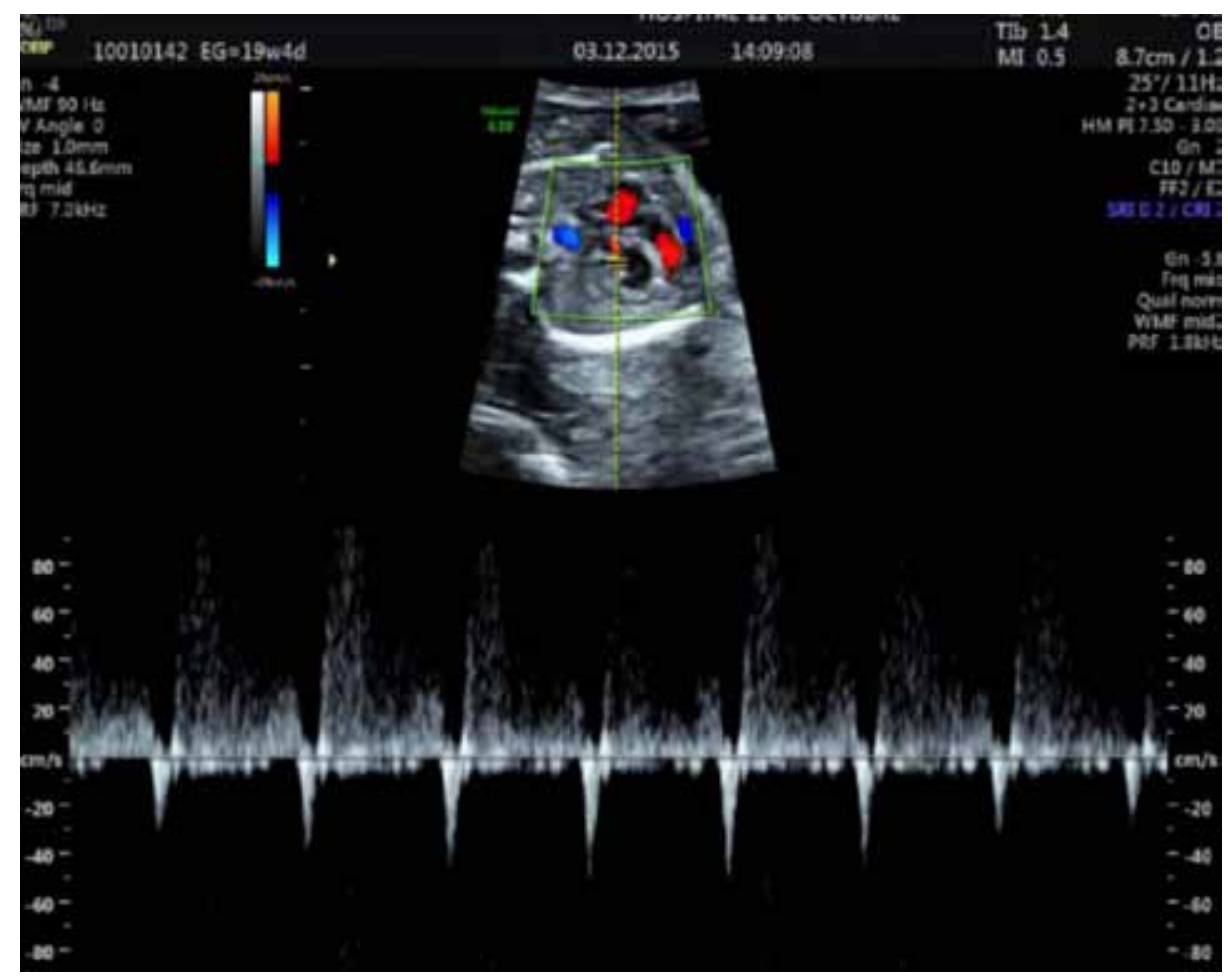

Figura 2. Llenado mitral monofásico.

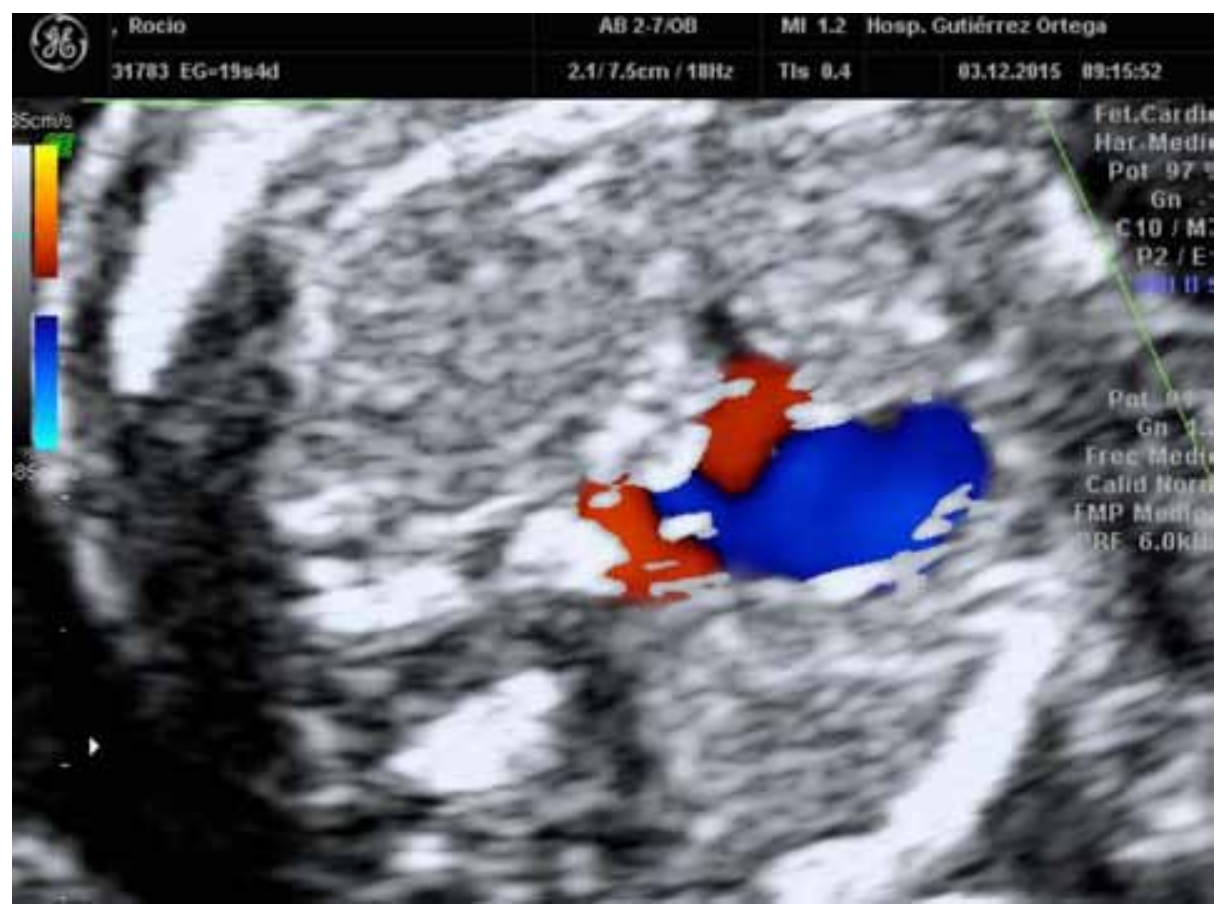

Figura 3. Arco aórtico hipoplásico y relleno retrogrado por el ductus. 


\section{DISCUSIÓN}

EI SCIH agrupa a un conjunto de malformaciones cardíacas caracterizadas por atresia, estenosis o hipoplasia de la válvula aórtica, mitral o ambas, asociado a hipoplasia del arco aórtico. La obstrucción severa de las válvula semilunar ocasiona un importante aumento de las presión en el ventrículo que induce la proliferación de fibrina y depósitos de colágeno en el subendocardio, causando un engrosamiento difuso, que a su vez provoca rigidez e hiperecogenicidad del mismo, generando una disfunción ventricular diastólica y sistólica severa (6), que progresivamente produce un menor llenado anterógrado del mismo y por tanto una falta de crecimiento ventricular que condicionará al recién nacido a la circulación univentricular $(5,7,8)$.

En cuanto al diagnóstico prenatal el empleo de ecografía morfológica en el segundo trimestre, que incluye la ecocardiografía fetal básica ampliada de 5 planos transversos, propuesta por Yagel y cols (1), alcanza unas tasas de detección en torno 60$80 \%$ de malformaciones cardíacas mayores $(5,9)$. Esto permite planificar el nacimiento y ofrecer un tratamiento precoz, reduciendo significativamente la morbimortalidad de algunas cardiopatías congénitas (9).

En nuestro caso el diagnóstico precoz de este tipo de patología demuestra que el entrenamiento en ecocardiografía fetal mejora las tasas de detección, lo que permite la derivación de los mismos a centros terciarios especializados donde puedan realizar terapias en pacientes seleccionados. En los últimos años la introducción del intervencionismo en patología cardíaca fetal, ha despertado un interés enorme por tratar de modificar la historia natural de esta enfermedad que permitan lograr una circulación biventricular y una cirugía correctora postnatal (10). La valvuloplastia intrauterina con balón aórtico (3) debe realizarse en pacientes seleccionados basándose en criterios cardiométricos (11) específicos, que pretenden determinar la situación hemodinámica fetal y la predicción de crecimiento y posible recuperación del ventrículo izquierdo disfuncionante.

La experiencia acumulada de este tipo de intervencionismo es todavía escasa debido a la poca incidencia de cardiopatías congénitas candidatas, el retraso en el diagnóstico prenatal y la falta de centralización de casos en un único centro que permita el entrenamiento técnico que el procedimiento necesita (11). A nivel mundial, los centros que acumulan mayor experiencia en este tipo de intervencionismo apenas superan el centenar de casos. Se han establecido diferentes scores para la selección adecuada de pacientes. El grupo de Boston (12) describió un sistema de puntuación pronóstico que otorga 1 punto a cada variable. Una puntuación $>4$ significa que la posibilidad de lograr una circulación biventricular alcanza el $42 \%$, mientras que si dicha puntuación es < 4 su tasa es del 0\% $(11,13)$. Existen scores que permiten predecir la progresión a un $\mathrm{SCIH}$, identificando fetos con formas intermedias de estenosis aórticas quienes conservarían una circulación biventricular aún sin recibir terapia prenatal (3).

En nuestro caso, la paciente no era candidata a valvuloplastía aórtica fetal por presentar un ventrículo izquierdo no rescatable. Al nacimiento, la paliación secuencial de la cardiopatía congénita consistiría en la realización de al menos tres cirugías en distinto tiempo que tienen como objetivo convertir el ventrículo derecho en el ventrículo sistémico (Norwood), y obviar el paso del retorno venoso sistémico por el corazón, siendo redirigido directamente a los pulmones (Glen-Fontan) (5). La mortalidad global de esta cardiopatía se sitúa en torno al $30-40 \%$, y la morbilidad es elevada producto tanto de la propia complejidad de la cardiopatía como del tratamiento quirúrgico. Tras ofrecer dicha información, los padres decidieron acogerse a la interrupción voluntaria del embarazo por malformación fetal grave contemplada en la ley orgánica 2/210, en España.

\section{CONCLUSIONES}

Debido a que las cardiopatías congénitas son malformaciones evolutivas, la estenosis valvular aórtica puede ocasionar hipoplasia progresiva de las estructuras del corazón izquierdo siendo su detección precoz clave para poder cambiar el curso de la enfermedad. Así mismo, es fundamental el constante entrenamiento en ecocardiografía fetal de todos los profesionales dedicados al seguimiento de las gestantes. Por otro lado, la focalización de los casos seleccionados en determinados centros terciarios permitiría no solo perfeccionar sus técnicas sino que mejorarían considerablemente sus tasas de éxito biológico.

\section{REFERENCIAS}

1. Yagel S, Cohen SM, Achiron R. Examination of the fetal heart by five short-axis views: a proposed screening method for comprehensive cardiac evaluation. Ultrasound Obstet Gynecol 2001;17:367-9.

2. Thiene G, Frescura C. Anatomical and pathophysiological classification of congenital heart disease. Cardiovasc Pathol 2010;19:259-74.

3. Hoffman J, Kaplan S. The incidence of congenital heart disease. J Am Coll Cardiol 2002;39(12):18901900.

4. Hunter LE, Chubb H, Miller O, Sharland G, Simpson JM. Fetal aortic valve stenosis: a critique of case selection criteria for fetal intervention. Prenat Diagn 2015;35(12):1176-81.

5. Escribano D, Herraiz I, Galindo A. Defectos del corazón izquierdo. Galindo A, Gratacos E, Martinez J. 
Cardiología Fetal. 1era edición: Editorial Marbán. Madrid- España. 2015; pp 288-316.

6. Antiñoloa G, Manganoa Al, Morunob A, Gruesoc J, Bedoya JM. Diagnóstico prenatal de estenosis aórtica crítica y fibroelastosis endocárdica. Prog Obstet Ginecol 2005;48(6):299-302.

7. Marshall A. Hypoplastic left heart syndrome. Uptodate [internet]. 2015. [Acceso diciembre 2015]. Disponible en http://www.uptodate.com.

8. Luis García M, Izquierdo Riezu MA, Pastor Menchaca EJ. (2010). Estenosis aortica valvular, subvalvular y supravalvular Protocolos Diagnósticos y Terapéuticos en Cardiología Pediátrica 2010. Capítulo 21. pp 271292. Disponible en http://www.secardioped.org/

9. Sainz JA, Zurita MJ, Guillen I, Borrero C, García-Mejido J, Almeida C, et al. Prenatal screening of congenital heart defects in population al low risk of congenital defects. A reality today. An Peditr (Barc) 2015;82:2734.
10. Laranjo S, Costa G, Freitas I, Ferreira JD, Bakero $\mathrm{L}$, Trigo $\mathrm{C}$, et al. The hybrid aproach for palliation of hypoplastic left heart syndrome: Intermediate result of a single-center experience. Rev Port Cardiol 2015;34(5):347-55.

11. Escribano D, Herraiz I, Mendoza A, Galindo A. Intervencionismo cardíaco fetal. Diagn Prenat 2012;23(4):137-47.

12. Marantz $P$, Grinenco $S$. Fetal intervention for critical aortic stenosis: advances, research and postnatal follow up. Curr Opin Cardiol 2015,30(1):89-94.

13. Tworetzky W, Wilkins-Haug $L$, Jennings $R$, Van der Velde ME, Marshall AC, Marx GR, et al. Balloon dilation of severe aortic stenosis in the fetus potential for prevention of hypoplastic left heart syndrome candidate selection, technique, and results of successful intervention. Circulation 2004;110(15):2125-31. 